\title{
An improved Synchronization signal detection algorithm in LTE 230
}

\author{
Chengling Jiang ${ }^{1,}$, Ping $\mathrm{Jia}^{1}$, Bo guo ${ }^{1}$ and Tao Wang $^{2, \mathrm{~b}}$ \\ ${ }^{1}$ State Grid Jiangsu Electric Power Company Information \& Telecommunication Branch, Nanjing \\ 210094, China; \\ ${ }^{2}$ School of Electronic and Optical Engineering, Nanjing University of Science and Technology, \\ Nanjing 210094, China. \\ ${ }^{a}$ chengling_jiang@163.com, bontor@126.com
}

Keywords: synchronization, frequency offset, PSS

\begin{abstract}
LTE 230 system is designed for the smart grid to transmit information in a wireless method instead of the wired or manual method. PSS (Primary synchronization signal) is the synchronization signal first detected in LTE 230 system. The timing error caused by PSS will have a ripple effect on the demodulation of downlink signal and the transmission of uplink signal. The detection of PSS is usually operated in time domain (i.e., time domain correlation) under the assumption of perfect timing. However, although little frequency offset will be introduced due to the terminals are fixed; the local oscillators of LTE 230 do not have a high precision considering the cost factor. An improved detection algorithm is proposed to amend the deviation caused by frequency offset.
\end{abstract}

\section{Introduction}

An electric power system cell search procedure must be executed when an UE wants to access a LTE 230 cell belonging to the electric power system. After the cell search procedure containing a serial process of synchronization, UE can obtain the initial timing of the OFDM symbol, which is crucial to the demodulation of the downlink signal and the transmission of uplink signal needing precise timing.

In LTE 230 system, we have three requirements for synchronization [1]. The first one is to capture the actual initial timing of symbol. The second one is to achieve frequency synchronization by mitigating the frequency offset determined by the device frequency synthesizer error plus Doppler frequency. Finally, the synchronization of sampling clock is also necessary.

Primary and Secondary Synchronization Signals (PSS/SSS) designed explicitly for the timimg imformation capture used in LTE can be inherited in LTE 230 electric power system prfectly. A UE can solve the complete PCI by first locating PSS which has three possible waveforms, and then searching for_SSS within a much confined time interval provided by the rough timing of PSS [2]. The detection performance of PSS is obviously the bottleneck of the timing accuracy, so this paper will focus on the detection of PSS to reduce the timing error at the origin. A commom detection method is to do time domain correlation and locate to the peak value timing ignoring the frequency offset effect due to the UE is ussaully fix (i.e., no relative mobility). Unfortunately, the UEs installed by the state grid is not feasible to be eqquipped with the high quality hardware as in a cellphone. So the frequency offset resulted from frequency mismatch in local ossilators between the transmitter and the receiver.the frequency deviation will be transformed into the timing detection error which has been proved in [3]. Obviouly, the deviation caused by frequency offset is not irreducible provided a frequency offset estimation is added.

The remainder of this paper is organized in follows. We first give an overview of the PSS and the SSS. Then we briefly describe the baseline PSS detection algorithm. finnally, we give a improved detection method to compete the freqeuncy offset effect and a comparison simulation results to show the performance gain. 

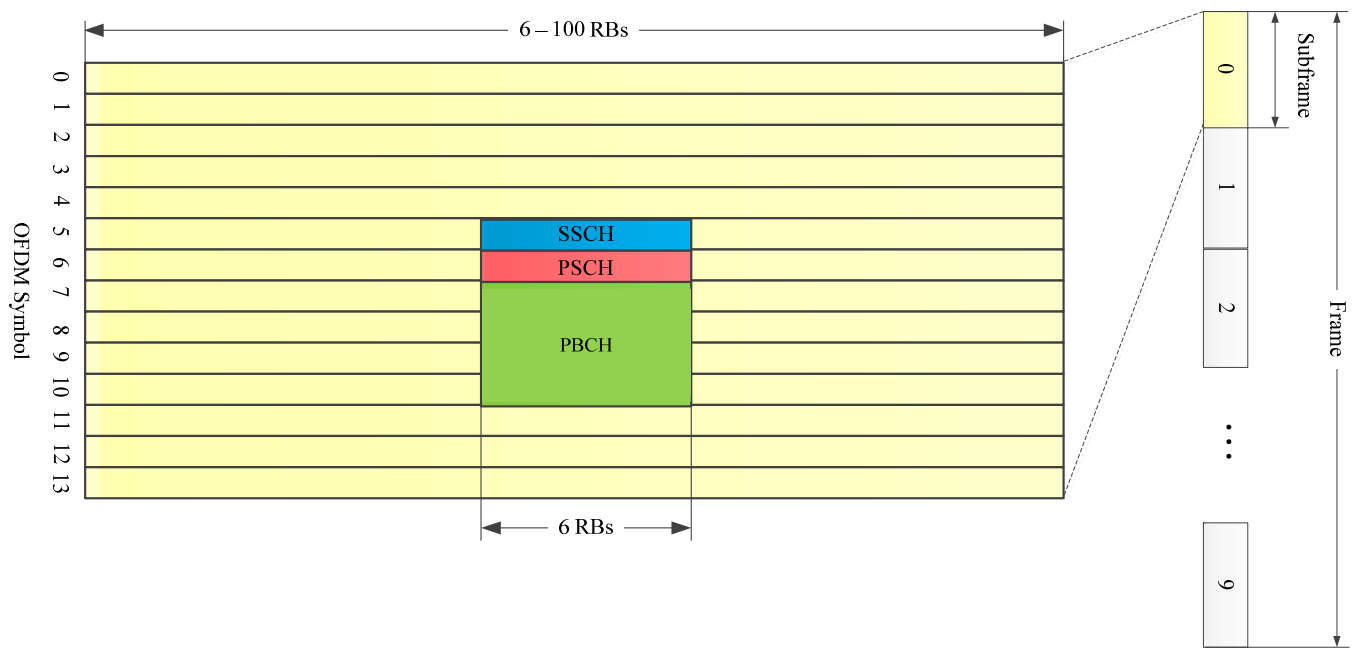

Figure 1 PSS/SSS structure

\section{An overview of PSS and SSS}

The PSS and the SSS are transmitted periodically on the last and second to last OFDM symbols of slot 0 of the first and the sixth subframes within a radio frame in frequency division duplex mode [4]. As shown in Figure 1, the PSS/SSS transmission is limited to the central $1.08 \mathrm{MHz}$ of the system bandwidth, even for a system whose actual bandwidth is larger than $1.08 \mathrm{MHz}$.

PSS/SSS (a synchronization signal) is meant to provide cell-specific timing; the number of synchronization waveforms has to be at least equal to the number of cell IDs. For example, we assume there are also 504 Physical cell IDs (PCIs) supported in LTE 230 system like in LTE, which is

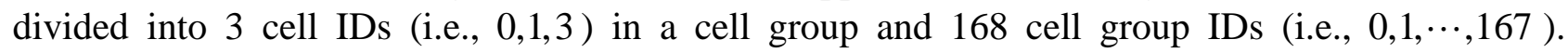
Consequently, a combination of $3 \times 168=504$ PCIs (i.e., $0,1, \cdots, 503$ ) are derived.

\section{Baseline detection algorithm}

The PSS is constructed from a frequency-domain ZC sequence of length 63 [5], with the middle element (i.e., ) punctured to avoid transmitting on the DC subcarrier. The sequency can be represented

as

$$
Z_{\mu}[i]=\exp \left[-\mathrm{j} \frac{\pi \mu n(\mathrm{n}+1)}{63}\right], \quad i=0,1, \cdots, 62
$$

Where $\mu$ is set to be $29,34,25$ for a better periodical outocorrelation and crosscorrelation, in addition to a relatively lower frequecy offset sensitivity [6].

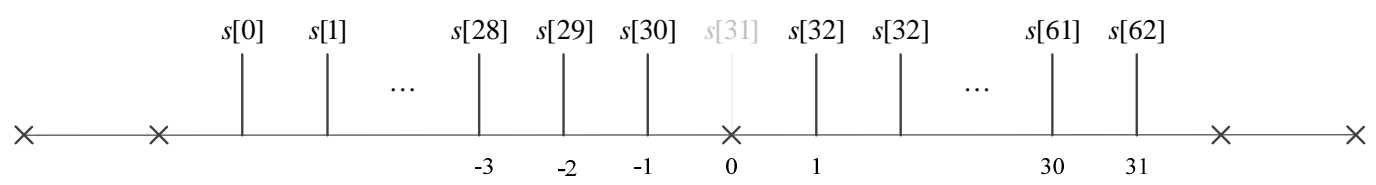

At the receiver, the output of the correlator (i.e., correlate the received signal samples $y$ with the conjugate of PSS sequence $s_{\mu}$ (the length is assumed to be $N$ ) in time domain) at hypothesis $k$ and root $\mu$ can be written as

$$
z[m]=\sum_{i=0}^{N-1} y[i+k] \bar{s}_{\mu}^{*}[i]
$$


Finally, we find the maximum correlation value among all, and determine the timing as PSS symbol timing; the root as the PSS sequence root.

Table 1 A concrete simulation results under the condition of different root and different frequency offset

\begin{tabular}{|c|c|c|c|c|c|c|}
\hline \multicolumn{7}{|c|}{ Timing error with frequency offset 0} \\
\hline SNR $(\mathrm{dB})$ & \multicolumn{2}{|c|}{ Root $=25$} & \multicolumn{2}{|c|}{ Root $=29$} & \multicolumn{2}{|c|}{ Root $=34$} \\
\hline & $\begin{array}{c}\text { Baselin } \\
\mathrm{e}\end{array}$ & Improved & $\begin{array}{c}\text { Baselin } \\
\mathrm{e}\end{array}$ & Improved & $\begin{array}{c}\text { Baselin } \\
\mathrm{e}\end{array}$ & Improved \\
\hline-5 & 0.0177 & 0.018 & 0.0158 & 0.016 & 0.017 & 0.0166 \\
\hline-2 & $<10^{-4}$ & $<10^{-4}$ & $<10^{-4}$ & $<10^{-4}$ & $<10^{-4}$ & $<10^{-4}$ \\
\hline \multicolumn{7}{|c|}{ Timing error with frequency offset $10 \mathrm{KHz}$} \\
\hline SNR (dB) & \multicolumn{2}{|c|}{ Root $=25$} & \multicolumn{2}{|c|}{ Root $=29$} & \multicolumn{2}{|c|}{ Root=34 } \\
\hline & $\begin{array}{c}\text { Baselin } \\
\mathrm{e}\end{array}$ & Improved & $\begin{array}{c}\text { Baselin } \\
\text { e }\end{array}$ & Improved & $\begin{array}{l}\text { Baselin } \\
\mathrm{e}\end{array}$ & Improved \\
\hline-5 & 0.1782 & 0.06 & 0.1561 & 0.0694 & 0.1659 & 0.0652 \\
\hline 0 & 0.0083 & $<10^{-4}$ & 0.0021 & $2 \times 10^{-4}$ & 0.0022 & 0.0014 \\
\hline 5 & $<10^{-4}$ & $<10^{-4}$ & $<10^{-4}$ & $<10^{-4}$ & $<10^{-4}$ & $<10^{-4}$ \\
\hline \multicolumn{7}{|c|}{ Timing error with frequency offset $13 \mathrm{KHz}$} \\
\hline SNR $(\mathrm{dB})$ & \multicolumn{2}{|c|}{ Root $=25$} & \multicolumn{2}{|c|}{ Root $=29$} & \multicolumn{2}{|c|}{ Root $=34$} \\
\hline & $\begin{array}{c}\text { Baselin } \\
\mathrm{e}\end{array}$ & Improved & $\begin{array}{c}\text { Baselin } \\
\text { e }\end{array}$ & Improved & $\begin{array}{c}\text { Baselin } \\
\mathrm{e}\end{array}$ & Improved \\
\hline-5 & 0.448 & 0.1837 & 0.3988 & 0.1875 & 0.411 & 0.1967 \\
\hline 0 & 0.1402 & $7.5 \times 10^{-4}$ & 0.0502 & 0.0018 & 0.06 & 0.0065 \\
\hline 5 & 0.0311 & $<10^{-4}$ & $7.5 \times 10^{-4}$ & $<10^{-4}$ & 0.001 & $<10^{-4}$ \\
\hline 10 & 0.0043 & $<10^{-4}$ & & & & \\
\hline
\end{tabular}

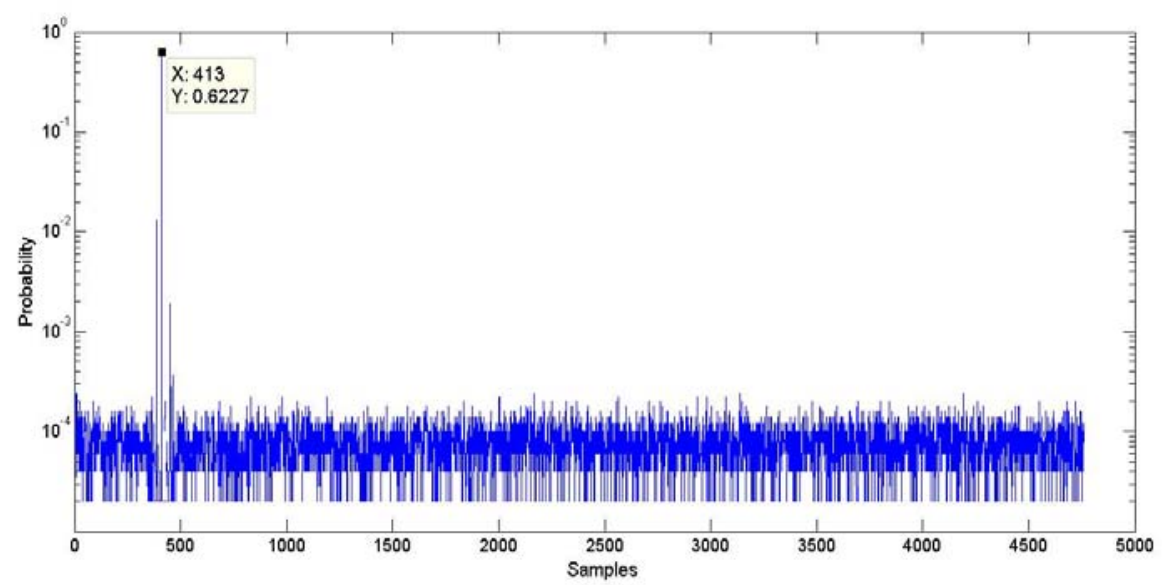

(a) Baseline detection probability

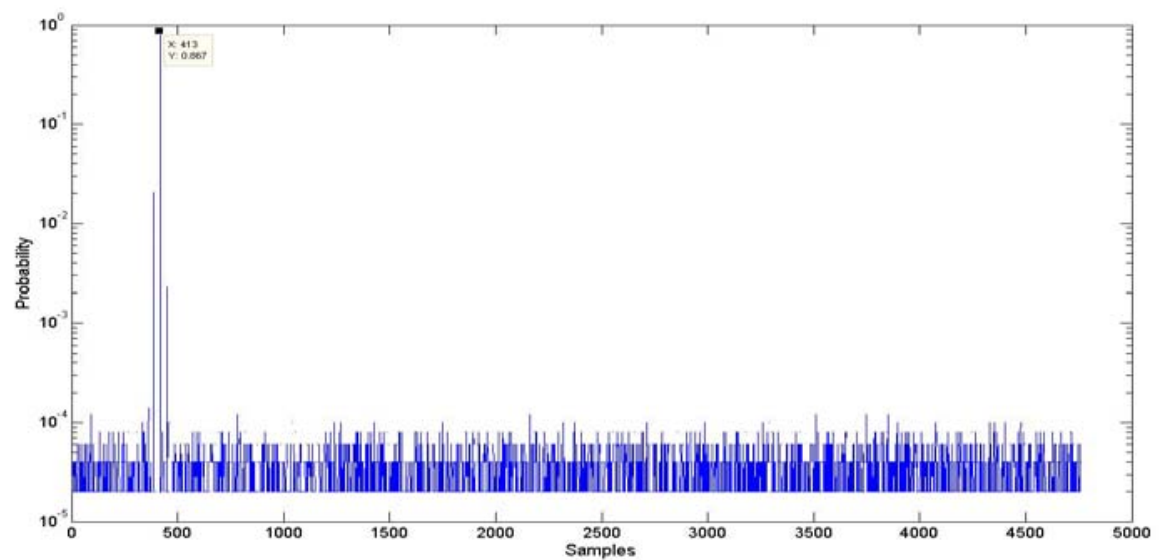

(b) Improved detection probabilitv

\section{Improved detection algorithm}


The improved detection aims to compensate the timing deviation caused by frequency offset. Then do the sum of correlations in $M$ groups each of which has $N / M$ samples at hypothesis $k$ and root $\mu$

$$
Z_{k, \mu}=\sum_{m=0}^{M-1} z[m]=\sum_{m=0}^{M-1}\left|\sum_{i=\frac{N}{M} m}^{\frac{N}{M}(m+1)-1} y[i+k] \bar{s}_{\mu}^{*}[i]\right|^{2}
$$

Then pick the combinations of timing hypothesis and root corresponding to the top $C$ correlation values. The frequency offset is estimated from the phase of the cross product between $z[m-1]$ and $z[m]$ for each candidate.

$$
\Delta f=\frac{M}{2 \pi N T_{s}} \arctan \sum_{m=1}^{M-1} z^{*}[m-1] z[m]
$$

Where $T_{s}$ denotes the sample interval.

When all the frequency offset value has been estimated we do frequency compensation for each candidate. Then the time domain correlation is operated again for those $C$ candidates under the revised frequency again. Now we can obtain the timing and root information from the candidate with the maximum correlation.

Compared simulation results are shown in Figure 2, we can see that at the frequency offset $10 \mathrm{KHz}$, the right timing detection probability has been increased from 0.4771 to 0.6227 . A concrete simulation results under the condition of different root and different frequency offset are shown in Table 1 . Note that by taking advantage of the frequency offset information implicitly carrier in the signal the improved detection performances bring a spectacle gain than baseline.

\section{Summary}

The realization of wireless communication using the LTE 230 in smart grid depends largely on the premise of synchronization. The existing synchronization signal detection algorithm do not provide a good enough timing detection performance. This paper presents an improved algorithm based on the original one by mitigating the frequency offset. Simulation results have proved its effectiveness.

\section{References}

[1] 3GPP, TR 22.951, Digital Cellular Telecommunications System (Phase 2+); Universal Mobile Telecommunications System (UMTS); LTE; Service Aspects and Requirements for Network Sharing, Release 11, version 11.0.0, October 2012.

[2] 3GPP, TR 36.872, Technical Specification Group Radio Access Network Small Cell Enhancements for E-UTRA and E-UTRAN Physical Layer Aspects, Release 12, version.12.1.0, Dec. 2013.

[3] 3GPP, TS 36.101, LTE; Evolved Universal Terrestrial Radio Access (E-UTRA); User Equipment (UE) Radio Transmission and Reception, Release 11, version 11.9.0, August 2014.

[4] Shen Changguo, Li Bin, Gao Yuliang, et al. The new technology for smart grid power electricity utilization [J]. Electrical Engineering, 2010(8): 11-15.

[5] C. Yang, et al., “Over-the-air signaling design for cellular networks,” IEEE Wireless Commun., pp. 120-129, Aug. 2014.Li Tongzhi.

[6] 3GPP, TS 36.331, LTE; Evolved Universal Terrestrial Radio Access (E-UTRA); Radio Resource Control (RRC); Protocol Specification, Release 11, version 11.8.0, July 2014. 